\title{
Unsteady Boundary Layer Flow of a Micropolar Fluid at a Two-Dimensional Stagnation Point on a Moving Wall
}

\author{
Seema Goyal ${ }^{1 *}$ \\ ${ }^{1}$ Department of Maths, Meerut College Meerut, UP \\ *Corresponding author: seemagoyal19@gmail.com
}

Received: 25.11.2021; Revised: 19.12.2021; Accepted: 28.12.2021

(C) Society for Himalayan Action Research and Development

\begin{abstract}
The problem of unsteady boundary layer flow of a micropolar fluid at a two-dimensional stagnation point on a moving wall when the free stream velocity and wall temperature vary arbitrarily with time has been studied. The governing partial differential equations were solved numerically by the Keller box method. The micropolar fluid flow model on a moving wall is capable of predicting the results which exhibit turbulent flow characteristics. Numerical results obtained for velocity, micro rotations and temperature distributions are shown graphically. The velocity distribution has been illustrated for several positive and negative values of the wall velocity. The skin friction, couple stress and transfer rate are found to be strongly dependent on the coupling parameter and time, however the effect of variation in the micro rotation parameter is visible appreciably in case of couple stress only.
\end{abstract}

Keywords - micropolar fluid, Boundary layer, stagnation point, moving wall, Keller box.

\section{Introduction}

A micropolar fluid is a fluent medium whose properties and behaviour are affected by the local motion of material particles in each of its volume elements. This theory gives rise to the concept of inertial spin, body movements, micro stress averages and stress moments which have no counterpart in classical fluid theories. Micropolar fluid has many practical applications like solidification of liquid crystals, animal blood, extrusion of polymer fluids, colloidal suspension, cooling of metallic plates. Eringen (1964) has given the theory of micropolar fluids. Eringen (1972) extended the theory to thermo micro fluids taking into account the effect of micro elements of fluids on both the kinematics and conduction of heat. Boundary layer flow in micropolar fluid has been studied by Wilson
(1970). Most problem of practical interest are of unsteady in nature. Gorla (1983) analyzed the thermal boundary layer equations for the flow of an incompressible micropolar fluid in the vicinity of a two-dimensional stagnation point on a moving wall. The boundary layers of an unsteady stagnation point flow in a nanofluid is studied by N. Bachok, et al. (2012). S. Dholey (2018) investigate the unsteady separated stagnation point flow of a power-law fluid over a moving plate. The unsteady incompressible boundary layer flow of a micropolar fluid at a stagnation point is obtained by Kumar and Nath (1984).

The present work is devoted to the solution of unsteady boundary layer flow of micropolar fluid at a two-dimensional stagnation point on a moving wall when the free stream velocity and 
wall temperature very arbitrarily with time. The boundary condition for microrotation is taken as suggested by Ahmadi (1976). The governing partial differential equations was solved numerically by Keller box method (1977).
Consider and unsteady, laminar, incompressible two dimensional micropolar fluid flow near a stagnation point on a moving wall. The wall temperature varies with time and the dissipation term is negligible at the stagnation point.

\section{Mathematical formulation}

The free stream velocity $u_{\varepsilon}$ which vary arbitrarily with time, may be expressed as

$$
u_{e}=\phi\left(t^{*}\right) \quad, \quad t^{*}=a t \quad, \quad a=\left(\frac{d u_{e}}{d x}\right)_{t^{*}=0}
$$

Where ' $a$ ' is a constant having the dimension (time) $)^{-1}$. Introducing the following non dimensional variables.

$$
\begin{array}{ccc}
\eta & =\left(\frac{a \rho}{\mu}\right)^{\frac{1}{2}} y, \quad v=u_{\theta} F^{\prime}\left(\eta, t^{*}\right)+V_{W} H\left(\eta, t^{*}\right), & v=-\left(\frac{a \rho}{\mu}\right)^{\frac{1}{2}} \phi\left(t^{*}\right) F\left(\eta, t^{*}\right), \\
v=a^{\frac{3}{2}}\left(\frac{\rho}{\mu}\right)^{\frac{1}{2}} x S\left(\eta, t^{*}\right)+V_{W}\left(\frac{a \rho}{\mu}\right)^{\frac{1}{2}} P\left(\eta, t^{*}\right), & \left(u_{\theta}\right)_{t=0}=a x,
\end{array}
$$

$$
T=T_{\infty}+\left(T_{w_{0}}-T_{\infty}\right) G\left(\eta, t^{*}\right), \quad\left(T_{W}-T_{\infty}\right) /\left(T_{w_{0}}-T_{\infty}\right)=\phi_{1}\left(t^{*}\right)
$$

The governing equations are

$$
\begin{aligned}
& (1+R) \phi F^{\prime \prime \prime}+\phi^{2} F F^{\prime \prime}+\phi^{2}\left(1-F^{\prime 2}\right)+\phi_{t^{*}}\left(1-F^{\prime}\right)-\phi F_{t^{\prime}}+R S^{\prime}=0 \\
& (1+R) H^{\prime \prime}+\phi F H^{\prime}-\phi H F^{\prime}-H_{t^{*}}+R P^{\prime}=0 \\
& \text { (3) } \\
& \text { (4) }
\end{aligned}
$$

$A P^{\prime \prime}+\phi F P^{\prime}-H S-P_{t^{*}}-A_{1}\left(2 P+H^{\prime}\right)=0$

(6)

$$
\operatorname{Pr}^{-1} \phi_{1} G^{\prime \prime}-\delta^{*} \phi_{1} S G^{\prime}+\phi \phi_{1} F G^{\prime}-\phi_{1} G_{t^{*}}-\phi_{1} t^{*} G=0
$$

$$
\text { where } R=\frac{k}{\mu}, A=\frac{y}{\mu j}, A_{1}=\frac{k}{a p j} \text { and } \delta^{*}=\frac{a_{1} a}{\mu C_{F}}
$$

$\delta^{*}$ is the micropolar heat conduction parameter. The boundary conditions are:

$$
\begin{array}{rllll}
\text { at } \eta=0 ; & F^{\prime}=0 & F=0 & H=1 & S=-2^{-1} F^{\prime \prime} \phi\left(t^{*}\right) \quad P=-2^{-1} H^{\prime}(0) \quad G=\phi_{1} \\
\text { as } \eta \rightarrow \infty ; & F^{\prime} \rightarrow 1 & H \rightarrow 0 & S \rightarrow 0 & P \rightarrow 0 \quad G \rightarrow 0
\end{array}
$$


The skin friction coefficient at the wall is given by

$$
c_{f}=\frac{2 \tau_{W}}{\left[\rho\left(u_{\theta}^{2}\right)_{t=0}\right]}=2\left(R_{\theta}\right)^{-1 / 2}\left[(1+R) F_{W}^{\prime \prime}+(1+R)\left(\frac{V_{W}}{u_{s}}\right) H_{W}^{\prime}+R S_{W}+R\left(\frac{V_{W}}{u_{s}}\right) P_{W}\right]
$$

Where the shear stress at the wall is given by

$$
\tau_{w}=\left[(\mu+k) \frac{\partial u}{\partial y}+k v\right]_{y=0}
$$

The couple stress coefficient is

$$
M=\frac{c_{m}}{\left|\rho\left(u_{\varepsilon}^{2}\right)_{t^{*}-0}\right|}=A\left[S_{W}^{\prime}+\left(\frac{V_{W}}{u_{\varepsilon}}\right) P_{W}^{\prime}\right]
$$

where

$$
c_{m}=\left(\frac{\gamma x}{\lambda}\right)\left(v_{y}\right)_{y=0} \text { is the couple stress at the wall. }
$$

The heat transfer coefficient in terms of Nusselt number is

$$
N u=\frac{\left(x q_{W}\right)}{K_{f}\left(T_{W_{0}}-T_{\infty}\right)}=\left(R e_{x}\right)^{1 / 2}\left(G_{W}^{\prime}+\beta S_{W}\right)
$$

where

$$
q_{W}=-\left[K_{f}\left(\frac{\partial T}{\partial y}\right)+\beta_{1}\left(\frac{\partial N}{\partial x}\right)\right]_{y=0}, \quad \beta=\beta_{1} \frac{a}{K_{f}\left(T_{W_{0}}-T_{\infty}\right)}
$$

$q_{W}$ being the rate of heat transfer from the wall and $\beta$ being the dimensionless form to the heat conduction parameter $\beta_{1}$.

Method of solution - In order to solve equation (3-7), Keller box method (1977) is used. A twodimensional non uniform net is formed taking one axis as $\eta$ and the other $\mathrm{t}^{*}$. A very fine spacing near the stagnation point has been selected to obtain accurate velocity, microrotation and temperature profiles. Each one of the eqns. (3-7) is solved separately by writing it as a system of first order differential eqns.

Results and Discussion - The numerical result for the velocity field, microrotation field and temperature are obtained for different values of
$\mathrm{R}$, the micropolar parameter, $\mathrm{A}$, the microrotation parameter $A_{1}$, the coupling parameter. The unsteady free stream velocity and wall temperature distribution considered here are respectively given by

$$
\begin{aligned}
& \phi\left(t^{*}\right)=1+\epsilon t^{*^{2}}, \\
& \phi_{1}\left(t^{*}\right)=1+\epsilon_{1} t^{*}
\end{aligned}
$$


The Keller box method is much better in view of

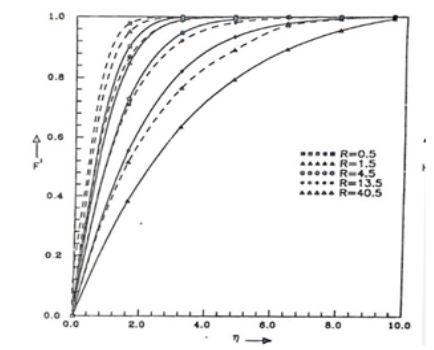

Fig.1 The velocity distribution $F^{\prime}$ for various values of $\mathrm{R}$ for $A_{1}=1.5, A=1.5, \varepsilon=0.5$

$-t^{*}=0, \ldots-t^{*}=1.5$

time and space taken. The velocity function $F$ and $\mathrm{H}$, the temperature distribution $\mathrm{G}$ become less steep as $\mathrm{R}$ increases but steepness increases with time as shown in fig. 1,2 and 7 . It is observed that the effect of microrotation parameter A is not much appreciable on $F^{\prime \prime}, \mathrm{H}$ and $G$ but the effect is more pronounced on microrotation function $\mathrm{S}$ and $\mathrm{P}($ fig. 4,6$)$ which also become more prominent with time. Fig.3 and fig. 5 also depict that with increase in $\mathrm{R}$ the microrotation increases near the plate but decreases further away from the plate.

From fig. 8 it may be observed that the ratio of streamwise velocity of the fluid to the wall velocity $\left(u_{\theta} / V_{W}\right)$ varies smoothly from a value of unity at the wall to the prescribed value of $\left(u_{\theta} / V_{W}\right)$ at the outer edge of the boundary layer for $t^{*}=0$. The results indicate that the velocity within the boundary layer cannot exceed the mainstream velocity. Fig.10-14 show the variation of skin friction coefficients $F_{W}^{\prime \prime}$ and $H_{W}^{\prime}$, microrotation gradients $S_{W}^{\prime}$ and $P_{W}^{\prime}$ and heat transfer coefficient $G_{W}^{\prime}$ for different values of R,
A and time $t^{*}$. All coefficients increase with $t^{*}$

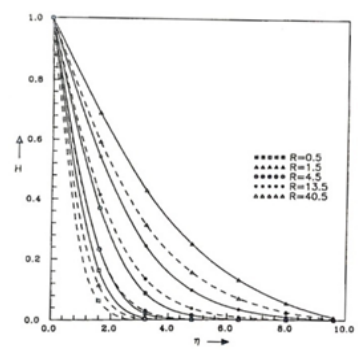

Fig.2 Distribution of $\mathrm{H}$ for various values of $\mathrm{R}$ for $A_{1}=1.5, A=1.5, \varepsilon=0.5$

$t^{*}=0, \ldots t^{*}=1.5$

whatever may be the values of parameter $\mathrm{R}$ and A. The reason for such an increase is that the fluid in the boundary layer is accelerated with time $t^{*}$ due to the continuous increase in the free stream velocity which in turn increases $F_{W}^{\prime \prime}, H_{W}^{\prime}$, $S_{W}^{\prime}, P_{W}^{\prime}, G_{W}^{\prime}$. In other words, the momentum boundary layer thickness, microrotation, thermal boundary layer thickness decreases with time $t^{*}$. It is observed that the effect of parameter $\mathrm{R}$ on the skin friction, microrotation gradient and heat transfer parameter is appreciable whereas the effect of parameter $\mathrm{A}$ is appreciable only on the microrotation gradient $S_{W}^{\prime}$ and $P_{W}^{\prime}$. These results are very much appropriate for the application of the micropolar fluid theory to suspension flows. Fig.15 illustrates the effect of Prandlt number Pr and the variation of wall temperature with time $\phi_{1}\left(t^{*}\right)=1+\epsilon_{1} t^{*}$ on the heat transfer parameter $G_{W}^{\prime}$. Large Prandlt numbers reduce the thermal boundary layer hence with the increase in $\operatorname{Pr}$, temperature gradient at the wall increases which cause a large surface heat transfer as shown in fig. 15 for all values of $t^{*}$. 


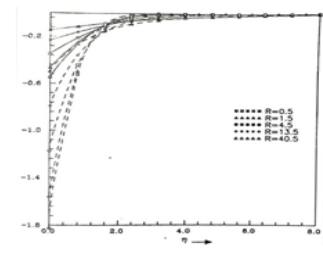

Fig. 3 Distribution of $\mathrm{S}$ for various values of $\mathrm{R}$ for $A_{1}=1.5, A=1.5, \varepsilon=0.5$ $t^{*}=0, \ldots-t^{*}=1.5$

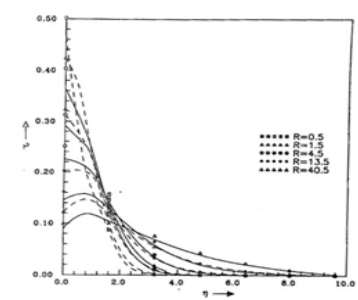

Fig. 5 Angular velocity distribution $P$ for various values of $\mathrm{R}$ for $A_{1}=1.5, A=1.5, \varepsilon=0.5$ $t^{*}=0,-t^{*}=1.5$

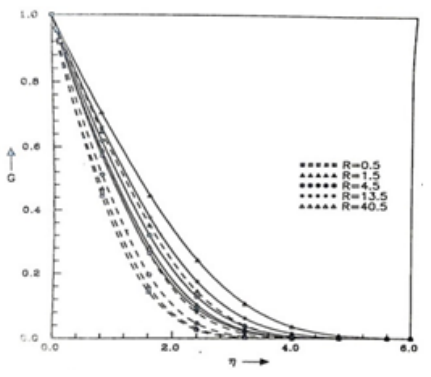

Fig.7 Temperature distribution for various values of $\mathrm{R}$ for $A_{1}=1.5, A=1.5, \varepsilon=0.5$

$t^{*}=0, \ldots t^{*}=1.5$

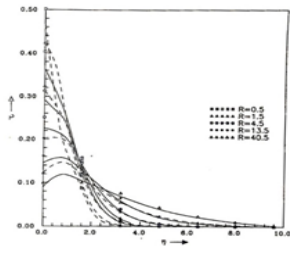

Fig.4 Distribution of $\mathrm{S}$ for various values of $\mathrm{A}$ for $A_{1}=1.5, R=1.5, \varepsilon=0.5$ $-t^{*}=0, \ldots-t^{*}=1.5$

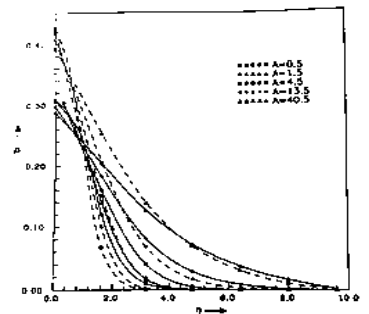

Fig.6 Angular velocity distribution $P$ for various values of $\mathrm{A}$ for $A_{1}=1.5, R=1.5, \varepsilon=0.5$ $t^{*}=0,-t^{*}=1.5$

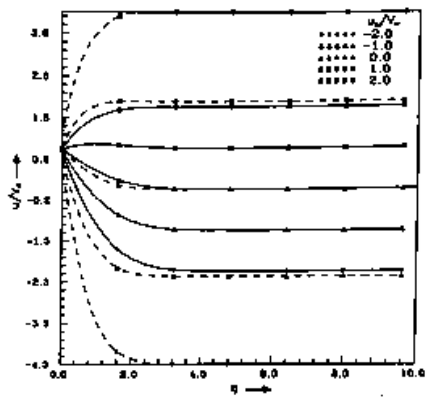

Fig. 8 Velocity distribution for various values of $\left(u_{\theta} / V_{W}\right)$ for $A_{1}=1.5, R=1.5, A=1.5, \varepsilon=0.5$ $t^{*}=0, \ldots t^{*}=1.5$ 


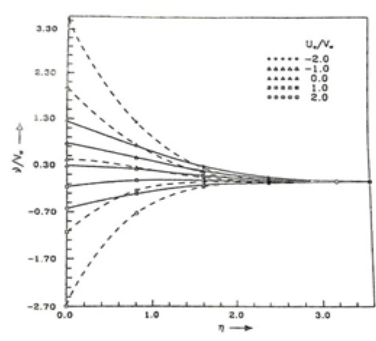

Fig.9 Angular velocity distribution for various values of $\left(u_{\theta} / V_{W}\right)$ for $A_{1}=1.5, R=1.5, A=1.5, \varepsilon=0.5$ $t^{*}=0, \ldots t^{*}=1.5$

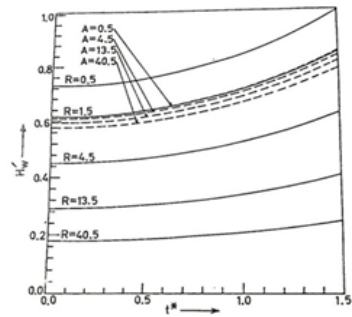

Fig.11 Skin friction parameter $H_{W}^{\prime}$ for various values of $\mathrm{R}$ and $\mathrm{A}$ for $A_{1}=1.5, \operatorname{Pr}=0.7, \varepsilon=0.5$

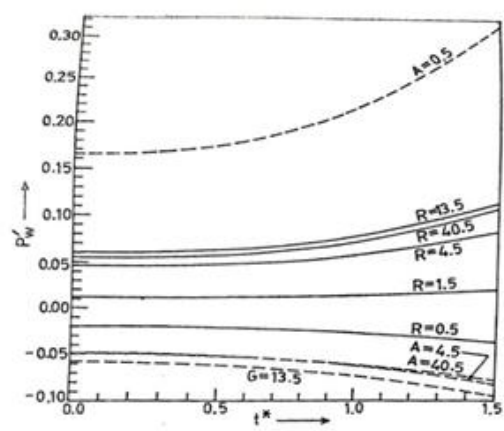

Fig.13 Micro rotation gradient parameter $P_{W}^{\prime}$ for various values of $\mathrm{R}$ and $\mathrm{A}$ for $A_{1}=1.5, \operatorname{Pr}=0.7, \varepsilon=0.5$

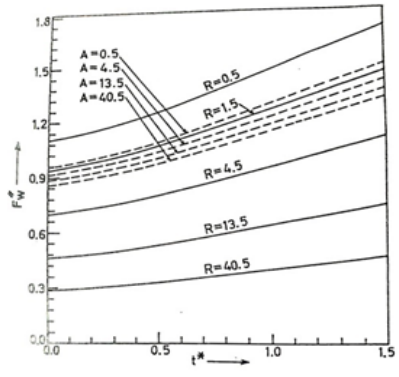

Fig. 10 Skin friction parameter $F_{W}^{\prime}$ for various values of $\mathrm{R}$ and $\mathrm{A}$ for $A_{1}=1.5, \operatorname{Pr}=0.7, \varepsilon=0.5$

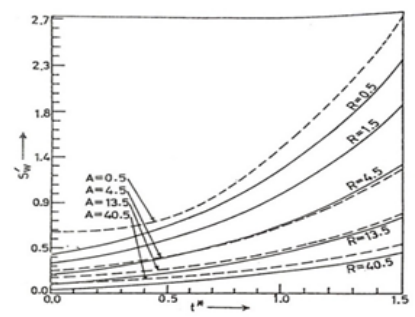

Fig.12 Micro rotation gradient parameter $S_{W}^{\prime}$ for various values of $\mathrm{R}$ and $\mathrm{A}$ for $A_{1}=1.5, \operatorname{Pr}=0.7, \varepsilon=0.5$

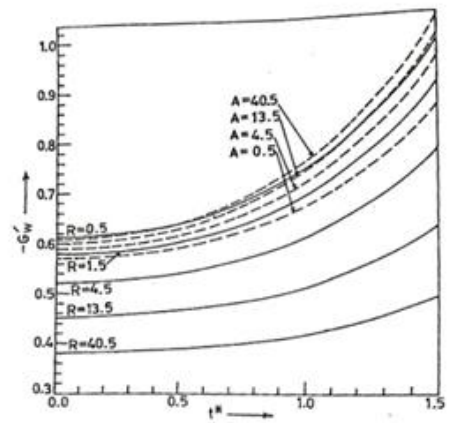

Fig. 14 Heat transfer parameter $-G_{W}^{\prime}$ for various values of $\mathrm{R}$ and $\mathrm{A}$ for $A_{1}=1.5, \operatorname{Pr}=0.7, \varepsilon=0.5$

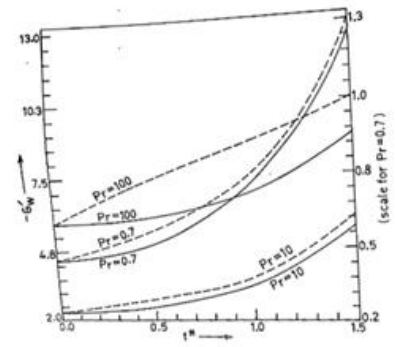

Fig. 15 Heat transfer parameter $-G_{W}^{\prime}$ for various values of $\operatorname{Pr}$ for $R=1.5, A=1.5, A_{1}=1.5, \varepsilon=0.5$ 


\section{References}

Ahmadi G, (1976) Self-similar solution of incompressible micropolar boundary layer flow over a semi-infinite plate , Int.J.Engng.Sci., 14,639.

Bachak N, Ishak A, Pop I, (2012) The boundary layers of an unsteady stagnation point flow in a nanofluid ,Int.J. of Heat and Mass Transfer, 55,6499.

Cebeci T, Bradshaw P (1977). Momentum transfer in boundary layer, Hemisphere, Washington,D.C.

Dholey S (2018) On the fluid dynamics of unsteady separated stagnation point flow of a power-law fluid on the surface of a moving flat plate, European J. of Mechanics-B/Fluids, 70,102.

Eringen AC (1964). Simple microfluid, Int.J.Engng.Sci.,2,205.

Eringen AC (1972) Theory of thermomicrofluids, J.Mat. Anal.Appl.,38,480.

Gorla R.S.R (1983) Micropolar boundary layer flow of a stagnation point on a moving wall, Int.J. Engng.Sci.,21,25.

Kumari M, Nath G (1984) Unsteady incompressible boundary layer flow of a micro polar fluid at a stagnation point ,Int.J.Engng.Sci., 22,755.

Willson A J (1972) Boundary layers in micropolarfluids, Proc. Camb. Phil.Soc., 67,469 . 